\title{
Visual Learning and Retention after Ablation of Inferotemporal Cortex in Dogs
}

By

\author{
Eiichi Iwai
}

From the Physiological Laboratory of Prof. K. Motokawa

(Received for publication, July 26, 1961)

\section{INTRODUCTION}

In recent years, considerable theoretical interest has been concentrated on the temporal lobe. Effects of bilateral ablation of temporal lobes on general behavioral changes in monkeys ${ }^{21}$, cats $^{21}$ and rats $^{20)}$ have been reported. The inferotemporal lobe syndrome by Klüver and $\mathrm{Bucy}^{83}$ in the rhesus monkeys is well-known and has frequently been cited.

More recently, studies on fractionized lesions in the temporal lobe of monkeys indicated that bitemporal lesion restricted to the surface on the inferior convexity of the temporal lobes was sufficient to produce visual deficits, but caused no apparent changes in affective behaviors $\left(\mathrm{Chow}^{3)}\right.$, Mishkin ${ }^{10)}$ ), while anteromedial temporal lesion caused affective changes rather than cognitive ones (Pribram \& Bagshaw ${ }^{18}$ ).

The visual disturbances produced by the bitemporal removal were attributed to the impairments of the so-called "higher visual center" (Chow', Mishkin \& Pribram ${ }^{12)}$, Pribram \& Mishkin ${ }^{19)}$ ) or the impairments of the primary visual process (Mishkin \& Hall ${ }^{11)}$, Pasik et al. ${ }^{16}$ ), but the nature of these deficits and underlying neural mechanisms are yet to be elucidated. In order to shed further light on this problem, it was attempted in the present experiments to investigate the effects of such ablation on the dog's performance capacity by determining thresholds of visual discrimination in which a modified method of a learning sets procedure by $\mathrm{Harlow}^{6}$ ) was used as a behavioral index of the intellectual performance capacity. It was investigated to what extent the learning capacity was impaired, and how the retention of what had previously been learned was after bilateral ablation of the inferotemporal lobes. In these experiments tests of different difficulties were used, for it seemed possible that the effects of ablation might be different depending upon the degree of difficulty.

The present experiments seem to provide further evidence that the bitemporal lesion produces the impairments of the so-called "higher visual center" 
rather than the primary sensory deficits.

\section{METHOD}

Subjects: Fifteen naive adult dogs were used. They had never been subjected to any kinds of conditioning before the present experiments.

Surgical procedures: After completion of the initial training, all subjects $\left(S_{S}\right)$ received a one-stage bilateral operation. $S_{S}$ were anesthetized with morphine $(10 \mathrm{mg} / \mathrm{kg})$ administered intramuscularly and with nэmbutal $(20 \mathrm{mg} / \mathrm{kg}$ ) administered intravenously. With aseptic technique, polus temporalis and gyrus temporalis inferior were symmetrically removed by subpial aspiration with use of a small gauge sucker (T-dog). In a control experiment, gyrus ectosylvius was bilaterally absorbed with the same surgical procedures as above (E-dog). The amount of ablated area in the E-dog was the same as in the T-dog. Some of T-dogs such as Dogs A, E and K were again subjected to ablation of the ectosylvian gyrus to make a control experiment, after all the experimental tests for the T-dog had been finished (T-E-dog). On the other hand, some of Edogs received an additional removal of the inferotemporal lobes (E-T-dog). Between the first and second operations, there was an interval of one to three months.

Apparatus and training procedures: Experiments were performed in a soundproof room dimly illuminated by a $20-\mathrm{W}$ lamp. Ss were gently restrained in a hammock, but could freely move their four limbs. The learning of the discrimination problem was established on the basis of instrumental conditioned avoidance responses. Ss learned to respond with a flexion of the leg to a positive conditioned stimulus (pos. CS), and not to respond to a negative CS. Both pos. and neg. CSs were presented for five soconds and the termination of pos. CS was overlapped with an unconditioned stimulus (US) lasting for two sec. Rhythmic electroshocks of 20 to $40 \mathrm{~V}$ were used as the US, which was strong enough to elicit a flexion of the leg. One of stimulating electrodes was a needle inserted into the left hind leg and the other was a tin plate on which the leg was placed. Thus, the US could be avoided by withdrawal of the leg in response to pos. CS. Two kinds of the problems, " $\alpha$ " and " $\beta$ ", were set in the present experiments. The problem $\alpha$ was more difficult and the stimuli in it consisted of flicker frequency (FF) of $7.5 \mathrm{cps}$ as pos. CS and that of $2.5 \mathrm{cps}$ as neg. CS. In the easier problem $\beta, \mathrm{FF}$ of $10 \mathrm{cps}$ as pos. CS and that of one cps as neg. CS were paired. Learning was said to have occurred when the level was attained at which more than 70 per cent correct responses were obtained to both pos. and neg. trials respectively over five consecutive days. If such a level was not attained within 1000 trials, no further trial was made. One session was run each day, consisting usually of 20 trials and balancing for pos. and neg. CSs. In each training session, pos. and neg. CSs were given in an entirely random order at intervals of 30 to 120 sec., 
in order to avoid temporal conditioning. Pre- and postoperative schedules contained overtraining ${ }^{7)}$, determination of the threshold of discrimination and retention tests in the pre- and postoperative periods in addition to initial training or retraining. Two separated experiments were conducted. In the first experiment, retention after a bitemporal lesion was investigated, and in the second, initial learning was studied in operated dogs. In the Experiment I, both problems $\alpha$ and $\beta$ were run. In the Experiment II, the problem $\alpha$ was not run because it seemed to be too difficult; even for T-dogs trained initially with the problem $\beta$ it was difficult to meet the criterion within the specified number of 1000 trials. After surgical intervention and five to seven days' recovery period, the postoperative relearning and the postoperative initial learning were made in the same way as mentionsd above. Before initiation of the formal training, all $S \mathrm{~s}$ were familialized with the experimental situations, but any kinds of preconditioning were not performed. Flickering light (FL) was produced by means of an electric stroboscope with a Xenon discharge tube, driven by a pulse generator.

Threshold of visual discrimination: After all the discrimination tests with the special pair of FFs (initial test) were finished, the capacity to discriminate between various FFs was tested at an interval of several days, paralleling with the overtraining course of the initial test. As the standard or comparison stimulus, the FF of $7.5 \mathrm{cps}$ in the dogs trained with the problem $\alpha$ or that of $10 \mathrm{cps}$ in the group trained with the problem $\beta$ was used. These stimuli were always pos. CS. The standard stimulus was paired with one of the lower frequencies such as 2.5, $3.0,3.5 \mathrm{cps}$, etc. in the dogs which learned the problem $\alpha$ or $1.0,1.5,2.0$, etc. in the dogs which learned the problem $\beta$, as the variable stimulus. The variable stimuli were always nogative in each pair and their frequency was raised in step of 0.5 , starting from 2.5 or $1.0 \mathrm{cps}$ until $S \mathrm{~s}$ could no longer discriminate. Such tests were always preceded by the initial test referred to above. For each pair of FFs about 20 trials were made, balancing for pos. and neg. stimuli and testing in the same way as in the initial tost. Several trials with the initial test problem were sometimes insorted to be sure that the dog's behavior to the test remained unchanged. Similar experiments were also performed after operation at various stages of relearning. When the operated dog failed in the initial test, the dog was subjected to easier tests, increasing the difference in frequency where the higher FF was fixed, but the lower neg. CS was decreased in frequency.

Informal observation of behavior: By two experimentors individually, detailed protocols were prepared concerning pre- and postoperative behavioral changes in the experimental situation and/or in the home house. Observation was made of spatial orientation, motor status, orality, səxuality, tameness or ferocity, maternal behavior, food intake, social dominance, etc. 


\section{RESULTS}

\section{Limitation of lesions}

After the experiments were finished, the animals were sacrificed with intravenous nembutal injection. The brains were perfused with 10 per cent formalin, embeded in celloidin and then serially sectioned at $40 \mu$ thickness. Frontal sections, stained with carbol-thionin, were examined macroscopically to identify lesions and also examined microscopically. Figs. 1 and 2 show the position and extent of the lesions in the Experiment I, together with representative frontal sections. Fig. 3 shows those of Experiment II. In these figures, ventral and lateral views are shown and lesions are indicated by black areas.

Generally speaking, the ablation conformed well to the experimental plan
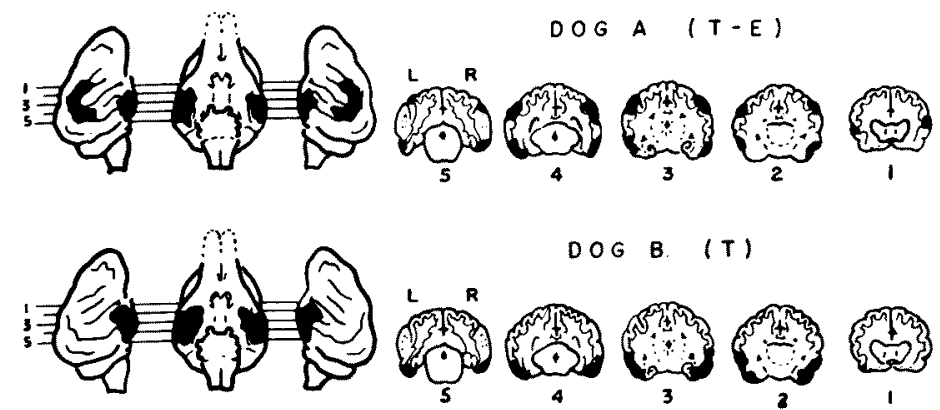

DOG B. (T)
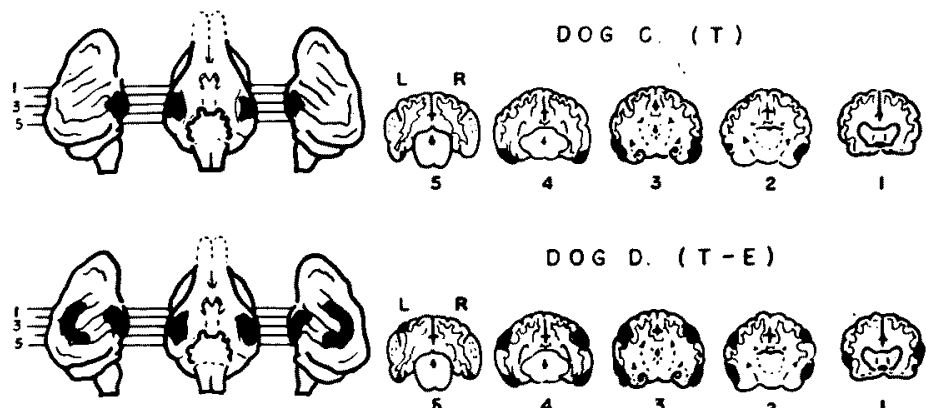

DOG D. $(T-E)$
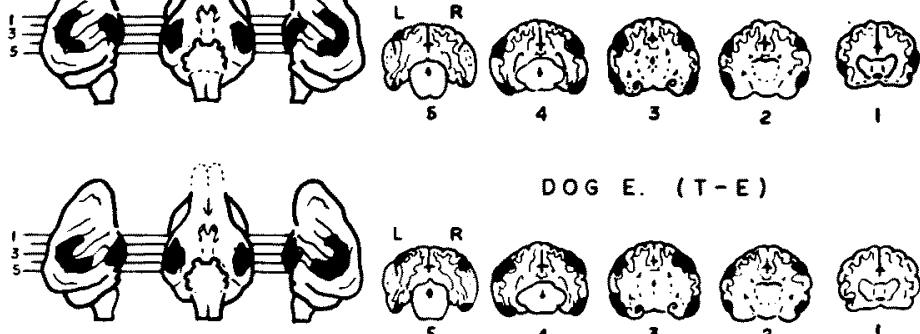

DOG E. $(T-E)$
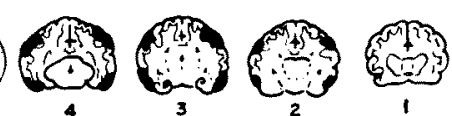

Fig. 1. Reconstructions of lesion in animals subjected to Experiment I on retention. Five representative frontal sections are shown to the right of lateral and ventral views of brain. Numbers for sections correspond to those given in lateral views. 


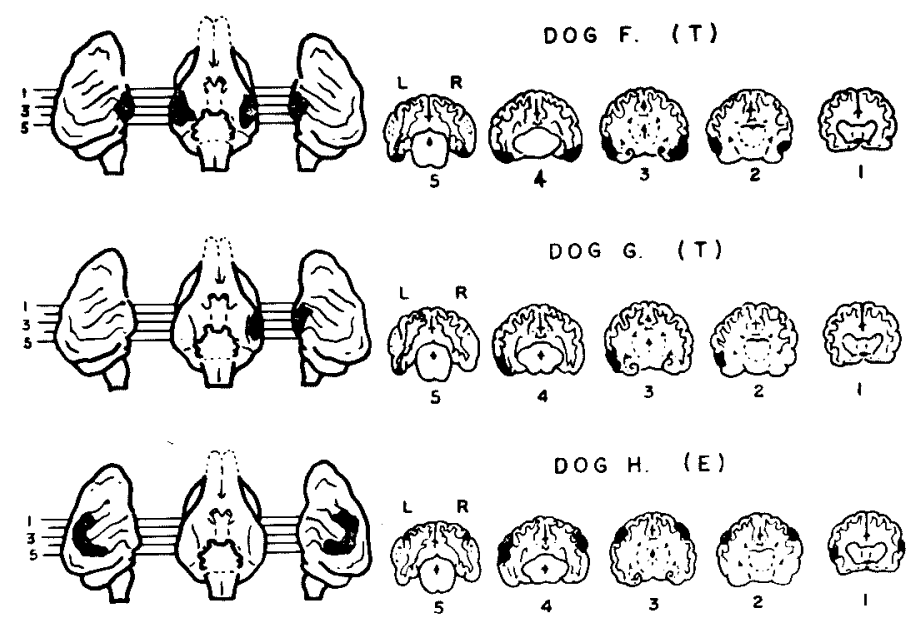

Fig. 2. Explanation as in Fig. 1.

in which the ablation of polus temporalis and gyrus temporalis inferior was aimed at. However, some relatively minor deviations were noted; an inferior portion of gyrus ectosylvius posterior or/and gyrus Sylvii posterior was more or less damaged in all casos, and the further lateral side of pyriformis was slightly invaded in some cases. Amygdala and hippocampus were entirely intact except for Dogs B, F and I. Dog B showed considerable deviation from the intended removal. In this dog, amygdala was largely removed in addition to the intended areas; $n$. basalis amygdalae parvicellularis and an inferior portion of $n$. lateralis amygdalae were bilaterally removed. $N$. cortalis amygdalae and $n$. basalis amygdalae magnocellularis were also invaded a little in the right hemisphere. An inferior portion of the right ventral hippocampus was also invaded slightly. The right hemisphere received more damage than the left did. Amygdala was almost safe in Dogs $\mathbf{F}$ and $\mathrm{J}$, although some of the lateral half of pyriformis was invaded, as shown in Figs. 2 and 3. Dog F received a lesion in an inferior portion of the ventral hippocampus in the right hemisphere. Dog I received slight destruction of an inferior portion of the right lateral amygdaloid nucleus. The ablation in Dog $\mathrm{C}$ was not sufficient ; sparing of a posterior portion of the bilateral inferior temporal gyri and sparing of medial side of the left temporal pole were observed.

In the control group, the removal was also in agreement with the intended areas of gyrus ectosylvius and an upper portion of gyri ectosylvius posterior and anterior, which belong to the auditory area. The amount of ablated area was the same as that of the inferotemporal lobe. Small deviation was also seen in the left hemisphere of Dog $M$; a little sparing of the ectosylvian gyrus and an invasion of the anterior Sylvian gyrus were seen.

The terminology used here to designate gyri, sulci as well as specific areas 

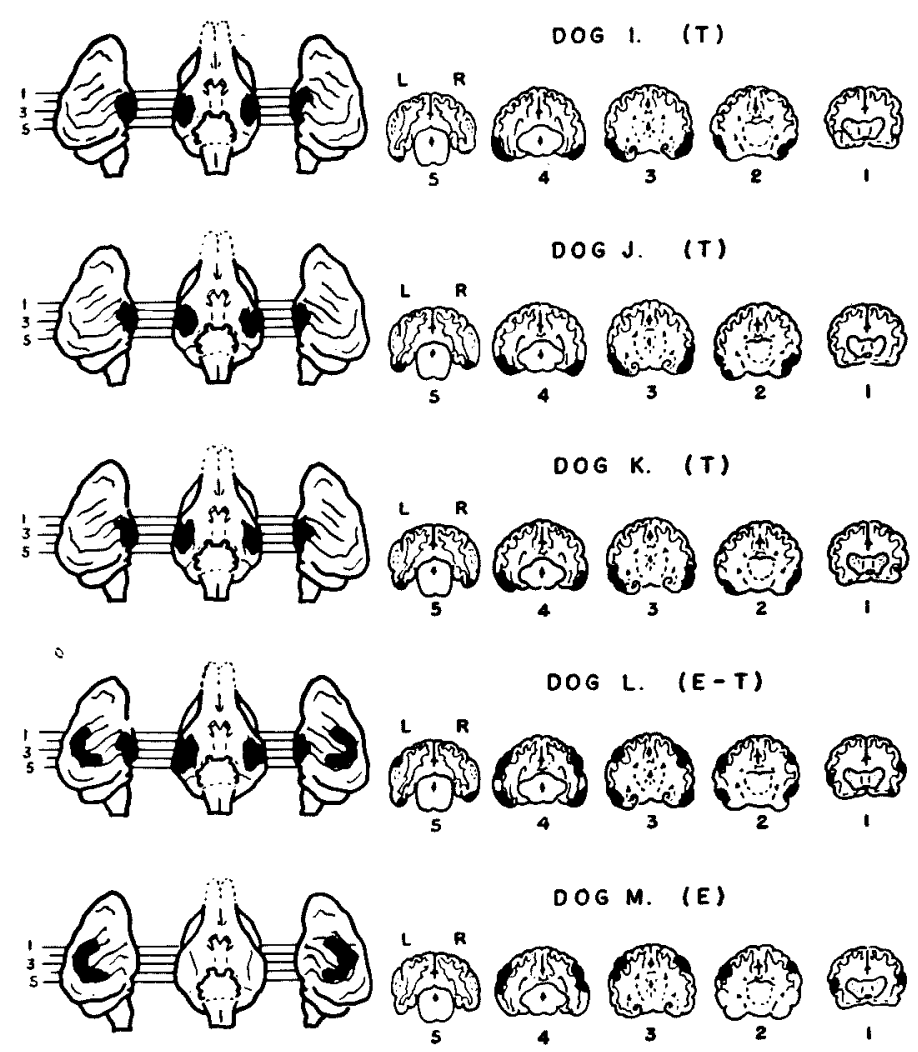

Fig. 3. Reconstructions of lesion in animals subjected to Experiment II on acquisition. Explanation as in Fig. 1.

was taken from "A Stereotaxic Atlas of the Dog's Brain" by Lim, Liu and Moffitt'.

2. Informal observation of behavior

a) General behaviors

Although detailed protocols were prepared, the T-dogs did not show any remarkable behavioral changes after operation. Neither ataxic symptoms nor gross visual disturbances could be observed. But in some cases temporary changes lasting for several days were observed; they represented an incomplete form of the Klüver-Bucy syndrome which consisted of visual agnosia, oral tendency, hypermetamorphosis and changes in emotional, sexual and dietary behaviors. The E-dogs did not show any kinds of the above-mentioned behavioral changes, but displayed some behaviors like sniffing.

An attractive episode was provided by Dog B from which a large part of amygdala in addition to the inferotemporal surface was bilaterally removed. 
Preoperatively, this dog had been pregnant and reared a litter of puppies successfully. After operation, the dog never wanted to breed hers and appeared to feel annoyed by them. This dog also displayed homosexuality and tended to get excited easily by trifles. This episode continued until this dog was sacrificed three months later.

\section{b) Spatial orientution}

The change in spatial orientation was the most marked and common one observed after operation. Before operation, Ss would rum back straight to their house when released from the test room. The T-dogs were different from normal in this behavior; they wandered about aimlessly and would not go home. They appeared, in general, less active and indifferent to surroundings, but they were capable of responding positively to anykinds of CSs with a flexion of the leg and making discrimination between pos. and neg. CSs to some extent.

On the contrary, the control dogs were not affected in orientation and could respond to experimentors after operation in the same manner as before operation.

\section{Experiment $I$}

a) Experimental group

It was attempted in this experiment to investigate retention and reacquisition of the visual performance after bilateral ablation of the inferotemporal cortex with special reference to the degree of difficulty of tests. Before operation, the number of trials necessary for attaining the criterion of learning was from 360 to 440 with the problem $\beta$ and more than 600 with the problem $\alpha$. Therefore, the problem $\alpha$ was more difficult than the problem $\beta$. Examples of learning curves with the problem $\alpha$ are shown in Fig. 4, in which percentage frequencies of CRs are plotted as ordinates against days of training as abscissae. Open circles denote positive responses to pos. CS and solid ones refer to positive responses to neg. CS. The greater the difference of both curves, the better discrimination. It was so defined that discrimination was successful when the positive responses to pos. CS was higher in occurrence than 70 per cent, while the positive responses to neg. CS was lower than 30 per cent. Such a state was preoperatively attained after 30 days during which 600 trials were made. After operation (lower diagram), learning capacity was so much affected that the criterion could not be reached even after 65 days or 1140 trials. In Fig. 5, learning curves of another $\operatorname{dog}(\operatorname{Dog} F)$ which was trained with the easier problem $\beta$ are shown. In this case, 18 days or 360 trials were enough to reach the criterion before operation, and 13 days or 260 trials were required to relearn after operation.

Problem $\alpha$ : Although eight dogs were conducted with the problem $\alpha$ prior to operation, only two of them succeeded in learning. Dog A got the criterion of the problem $\alpha$ after 900 trials preoperatively (4th column in Table I) and 

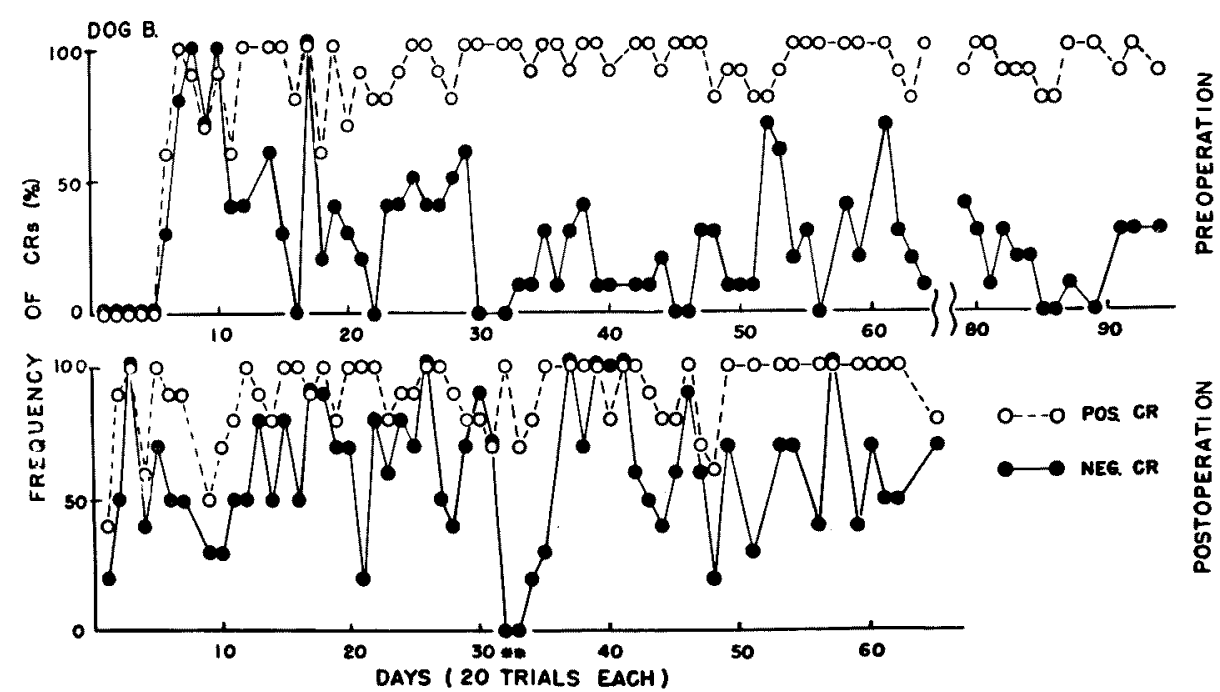

Fig. 4. Pre-(upper) and post- (lower) operative learning curves determined with a more difficult discrimination task $a$ (Dog B). Open circles denote percentage frequencies of positive conditioned responses to positive conditioned stimuli, and filled circles are those to negative conditioned stimuli. Asterisk $(*)$ denotes "educational effect" described in text.

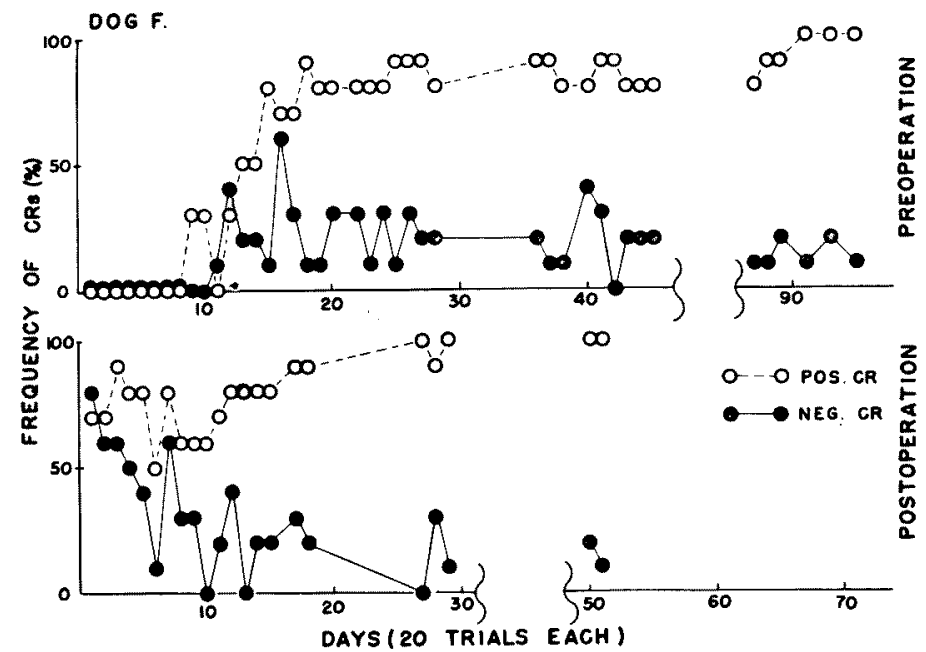

Fig. 5. Pre- and postoperative learning curves determined with an easier discrimination task $\beta(\operatorname{Dog} F)$. Explanation as in Fig. 4.

could relearn it within 960 trials postoperatively (7th column in Table I). In view of the fact that some retention was observed in this dog immediately after operation, the postoperative scores 960 , which were larger than the preoperative 
ones 900 , indicated that the learning capacity of this dog was affected by the operation. Another Dog B, which counted the number of 600 trials until the preoperative criterion was attained, failed to relearn the problem $\alpha$ after operation even with more than 1140 trials, twice the preoperative learning scores. Postoperatively, Dog B could easily performed the easier problem $\beta$ with which this dog had not been trained initially, whereas this $\operatorname{dog}$ could not reach the criterion in learning of the problem $\alpha$. Strange to say, a few trials of presentation of the easier problem $\beta$ prior to that of the problem $\alpha$ made this dog reach the criterion in learning of the problem $\alpha$. It seemed as if success in an easier task increased the discrimination capacity, though temporarily. The effect was called an "educational effect" in this paper. As shown in the postoperative learning curves of Fig. 4 (lower diagram), the learning was once established on the 32nd and 33rd days and this was due to the educational effect. Dog C could not meet the criterion within 1200 trials preoperatively, but discrimination as tested by the problem $\alpha$ seemed to be better than mere chance. After operation, discrimination capacity was entirely lost in such a manner that the dog showed only positive responses to all kinds of stimuli (stage of generalization).

Problem $\beta$ : Five dogs were subjected to tests with the problem $\beta$ and could easily learn them after 360 to 440 trials. The complete retention of the performance could not be seen right after operation, but all $S$ s could relearn the

TABLE I. Learning Scores of Visual Diserimination (Experimental Group of Experiment I)

\begin{tabular}{|c|c|c|c|c|c|c|c|c|}
\hline \multirow[b]{2}{*}{ Animal } & \multirow{2}{*}{$\frac{g}{0}$} & \multicolumn{4}{|c|}{ Preoperative } & \multicolumn{3}{|c|}{ Postoperative } \\
\hline & & $\begin{array}{l}\text { Trials } \\
\text { necessary } \\
\text { to cause } \\
\text { first CR } \\
\end{array}$ & $\begin{array}{l}\text { Initial } \\
\text { learning }\end{array}$ & Retention & $\begin{array}{l}\text { Threshold } \\
\left(\begin{array}{c}\text { cps of } \\
\text { neg. CR }\end{array}\right)\end{array}$ & $\begin{array}{l}\text { Relearn- } \\
\text { ing }\end{array}$ & Retention & $\left.\begin{array}{l}\text { Threshold } \\
(\text { cps of } \\
\text { neg. CS }\end{array}\right)$ \\
\hline Dog A & $\alpha$ & 140 & 880 & 0 & 4.0 & 940 & 0 & 1.0 \\
\hline $\operatorname{Dog} B$ & $\alpha$ & 100 & 580 & 20 & 4.0 & $1140 *$ & - & 1.0 \\
\hline Dog C & $a$ & 140 & $1200 *$ & - & - & $1020^{*}$ & - & - \\
\hline $\operatorname{Dog} \mathrm{D}$ & $\begin{array}{c}\alpha \\
\downarrow \\
\beta\end{array}$ & 180 & $\begin{array}{c}840^{*} \\
\downarrow \\
80\end{array}$ & 0 & 2.5 & 360 & 0 & 0.5 \\
\hline $\operatorname{Dog} E$ & $\beta$ & 180 & 420 & 0 & 4.0 & 60 & 0 & 3.0 \\
\hline $\operatorname{Dog} \mathbf{F}$ & $\beta$ & 160 & 340 & 0 & 3.0 & 240 & 0 & 0.5 \\
\hline $\operatorname{Dog} G$ & $\begin{array}{r}\alpha \\
\downarrow \\
\beta\end{array}$ & 220 & $\begin{array}{c}900 * \\
\downarrow \\
0\end{array}$ & 0 & 2.5 & 0 & 0 & 3.0 \\
\hline Dog L & $\beta$ & 120 & 300 & 0 & 3.5 & Died on & the way & f relearning \\
\hline
\end{tabular}

Note: Scores represented in above table are trials required to attain criterion.

Scores of threshold presented here are last preoperative values and first postoperative ones. Asterisk (*) denotes failure to acquire the habits within the specified number of training trials. Arrow indicates that the imposed problem " $a$ " was changed into " $\beta$ " after failure of learning on " $a$ ". Dashed line represents that dog was not tested at that stage. 
problem rather rapidly as shown in Table I (columns 4 and 7 ). The so rapid relearning in Dog $\mathbf{E}$, for which only 80 trials were sufficient, was probably due to the circumstance that preoperative overtraining ${ }^{7,13)}$ was more extensive in this dog than did in others. Fig. 6 shows how easy the problem $\beta$ was to relearn

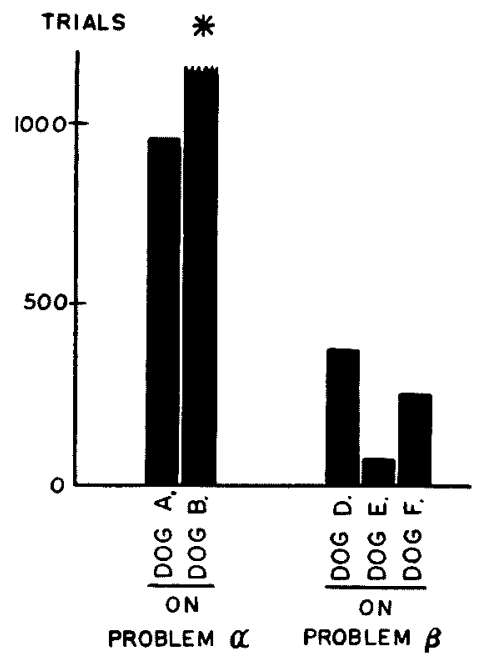

Fig. 6. Postoperative relearning scores (Experiment I on retention). Number of trials required to reach criterion is greater for group trained with more difficult problem $\alpha$ than for that with easier problem $\beta$. Asterisk $(*)$ denotes failure in reaching criterion.

postoperatively in comparison with the problem $a$. Dog $\mathrm{L}$, which had received operation in both ectosylvian gyri, showed a complete retention of the preoperative learning after operation. In the same dog, ablation of both inferotemporal lobes was carried out one and a half months after the first operation. After this second operation, a great deficit in visual performance was found, although it was impossible owing to the death of the dog to determine whether or not this dog would be able to relearn the problem in the long run. Dog G, which had received a left milateral lesion in the inferotemporal lobe, showed a complete retention.

It may be concluded from these findings that loss in learned visual performance was greator with the more difficult problem $\alpha$ than with the easier problem $\beta$.

b) Control group

Bilateral ablation of ectosylvian gyri: For control to the effects of bilateral ablation of the inferotemporal lobes upon the deficits of visual discrimination, gyrus ectosylvius which belongs to an auditory area was bilaterally ablated. The amount of ablated area was made almost the same as that of the inferotemporal lobe as shown in Figs. 1, 2 and 3. The results obtained are represented in Table II. The discrimination learned by preoperative learning was wellretained. The threshold of discrimination was found to be the same as before opera- 
TABLE II. Learing Scores of Visual Discrimination (Operated Control Group of Experiment I)

\begin{tabular}{|c|c|c|c|c|c|c|}
\hline \multirow{2}{*}{ Animal } & \multirow{2}{*}{$\frac{\frac{g}{0}}{\frac{0}{0}}$} & \multicolumn{2}{|c|}{ Preoperative } & & \multicolumn{2}{|l|}{ Postoperative } \\
\hline & & & Threshold & & & Threshold \\
\hline $\operatorname{Dog} A$ & $\alpha$ & 0 & 4.0 & 20 & 0 & 4.0 \\
\hline Dog E & $\alpha$ & 0 & 4.5 & 40 & 0 & 4.0 \\
\hline Dog K & $\alpha$ & 0 & 3.5 & \multicolumn{3}{|c|}{ Died after surgery } \\
\hline Dog $H$ & $\beta$ & 20 & 2.0 & 0 & 0 & 2.0 \\
\hline Dog D & $\beta$ & 0 & 2.0 & 0 & 0 & 2.0 \\
\hline $\operatorname{Dog} \quad G$ & $\beta$ & 0 & 2.5 & 0 & - & 3.0 \\
\hline
\end{tabular}

Note: Refer to TABLE I.

tion. The number of trials in preoperative overtraining in this series was made far less than that of the T-dogs in order to control the effects of overtraining ${ }^{7}$.

Unilateral ablation of inferotemporal lobe: In Dog $G$ which was subjected to a unilateral ablation of the left inferotemporal lobe, the complete retention of the performance was observed after operation. The threshold of the visual discrimination of FFs was found improved a little in this dog. No affective changes were observed, either. These findings might indicate that the unilateral resection of the infeortemporal lobe did not affect the cognitive and affective behaviors.

Retention tests: It took usually a week for recovery from operation. The reduction in performance capacity after operation may be due to a normal process of forgetting. In order to answer this question a series of retention tests was performed preoperatively as well as postoperatively. The results obtained are summarized in Table I (columns 5 and 8). As can be seen in this Table, the retention was complete before operation as well as after operation. It is to be noted that the retention test after operation was made after releaming.

\section{Experiment $I I$}

In Experiment I all dogs experienced learning preoperatively. In the Experiment II, no special procedure of learning was performed before operation to examine the effects of operation upon the initial postoperative learning without influence of any preoperatively acquired memory. The rate of acquisition could be expressed by two indicators. The one was the number of trials required from commencement of training to the first $\mathrm{CR}$ to any kinds of flickering lights, and the second was that required for completion of learning. The former indicator refers to the number of trials which will be needed until the dog becomes aware of the relation between CS and US. Three T-dogs and two E-dogs were used in this experiment.

The results are summarized in Fig. 7. As shown in the left diagram of this figure, the first indicator, i.e., the number of trials needed for the first $\mathrm{CR}$ was 
much greater for the T-dogs than for normal or non-operated dogs (N-dogs). The operated control dogs or E-dogs were the same as the normal ones in this behavior. The second indicator, i.e., trials-score needed to reach the criterion of learning was also much higher in the $\mathrm{T}$-dogs than in the normal dogs and the E-dogs (see the right diagram in Fig. 7).

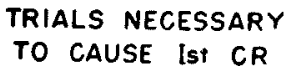

TRIALS NECESSARY
TO CAUSE IST CR
TRIALS TO REACH CRITERION

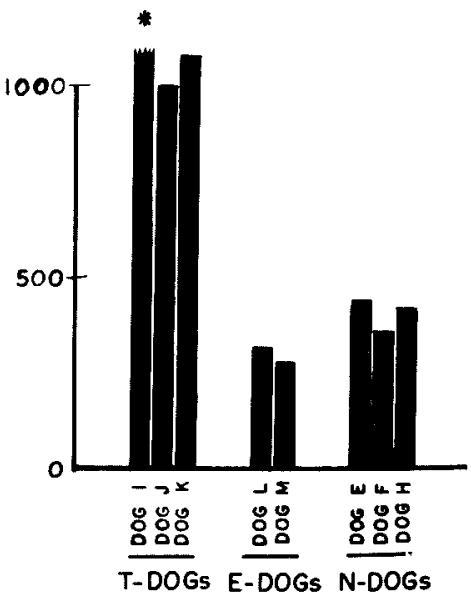

Fig. 7. Initial postoperative learning scores (Experiment II on acquisition). Columns in left figure denote trials required to cause first conditioned response and those in right indicate trials to reach criterion in learning. Temporal-operated dog: T-dog; Ectosylvius-operated dog: E-dog; Non-operated or normal dog: N-dog. Asterisk $(*)$ denotes failure in reaching criterion.

\section{Threshold of visual discrimination or differential threshold (DT).}

In order to investigate the manner of the pre- and postoperative performance capacity, the DT was determined in all dogs. The procedure used here was a modification of learning sets-learning-how-to-learn - procedure by Harlow ${ }^{6}$. A graded series of FFs was presented as neg. CSs, while the frequency of a pos. CS was fixed at a certain higher value.

When the difference in frequencies of pos. and neg. CSs was large enough, the dog showed a positive response to the pos. CS, but no response to the neg. one. As the difference decreased, the dog showed a positive response to the neg. CS more and more frequently. It was so defined that a differential threshold was attained when positive responses occurred to neg. CSs in 30 per cent of all trials with neg. CSs.

The changes in DT at pre- and postoperative stages are shown in Fig. 8. In this figure, the highest frequencies of the neg. CSs discriminated from the pos. CS were plotted as ordinates against the days of training as abscissae. Im- 
pairment and improvement of discrimination capacity are indicated by a fall and a rise of curve respectively. Dogs A and B were trained with the problem $\alpha$, difficult one. The other dogs $\mathrm{D}, \mathrm{F}$ and $\mathrm{E}$ were trained with the problem $\beta$, easier one. The highest possible frequency of neg. CS was $2.5-4.0 \mathrm{cps}$ at the preoperative stage (column 6 in Table I), but was found to be much lowered to $0.5-1.0 \mathrm{cps}$ about a week after bilateral ablation of the inferotemporal lobes (column 9 in Table I). This finding indicates that the discrimination capacity has strongly been affected by the operation. However, all dogs except Dog B showed complete recovery concerning the discrimination capacity after further training at the postoperative stage. After operation, the discrimination capacity was not so strongly reduced in Dog $\mathrm{E}$ as in the other dogs. This fact may be due to the circumstance that more intensive overtraining was carried out preoperatively in Dog $\mathrm{E}$ than did in the others ${ }^{7}$. Dog B, the exceptional case in which no recovery was seen, did not succeed in postoperative tests with the problem $a$ which was solved with success before operation, although this dog could make discrimination on the easier ones.

It is without saying that the postoperative discrimination capacity in the control dogs remained at the same level as at that before operation.

\section{DISCUSSION}

It has been shown above that marked impairments in visual discrimination of FFs were produced by bilateral ablation of the neocorticies on the inferior convexity of the temporal lobes, i.e., polus temporalis and gyrus temporalis inferior. Deficits in visual discrimination were revealed by three kinds of experiments on retention, acquisition and threshold of discrimination. Testing and training procedures and animal species used here were entirely different from those in the previous studies ${ }^{12,19)}$. Nevertheless, the results obtained in the present experiments are similar to those obtained from monkeys.

As to the mechanism of the impairments, there have been controversies; some investigators"12,10) postulated damages in the so-called "higher visual center", while others ${ }^{11,16)}$ assumed th! disturbances of the primary visual process. The latter hypothesis seems, however, unlikely for the following reasons. For example, Dog B could never solve the more difficult problem $\alpha$ without several preliminary trials with the easier problem $\beta$. It would be difficult to account for such an educational effect in terms of mere primary sensory deficits. Furthermore, it was directly revealed that the capacity to form learning sets which may indicate a behavioral degree of the intellectual performance was markedly impaired after inferotemporal operation. As has been shown above, the number of trials required to evoke the first CR was greater in the T-dogs than in the control dogs. This fact indicates that the mechanism for conditioning has been affected by operation. It is apparent that the central mechanism for conditioning 
is not at all restricted to the primary sensory mechanism. Judging from the behavior of the lexperimental animals, no gross defect was found in the visual

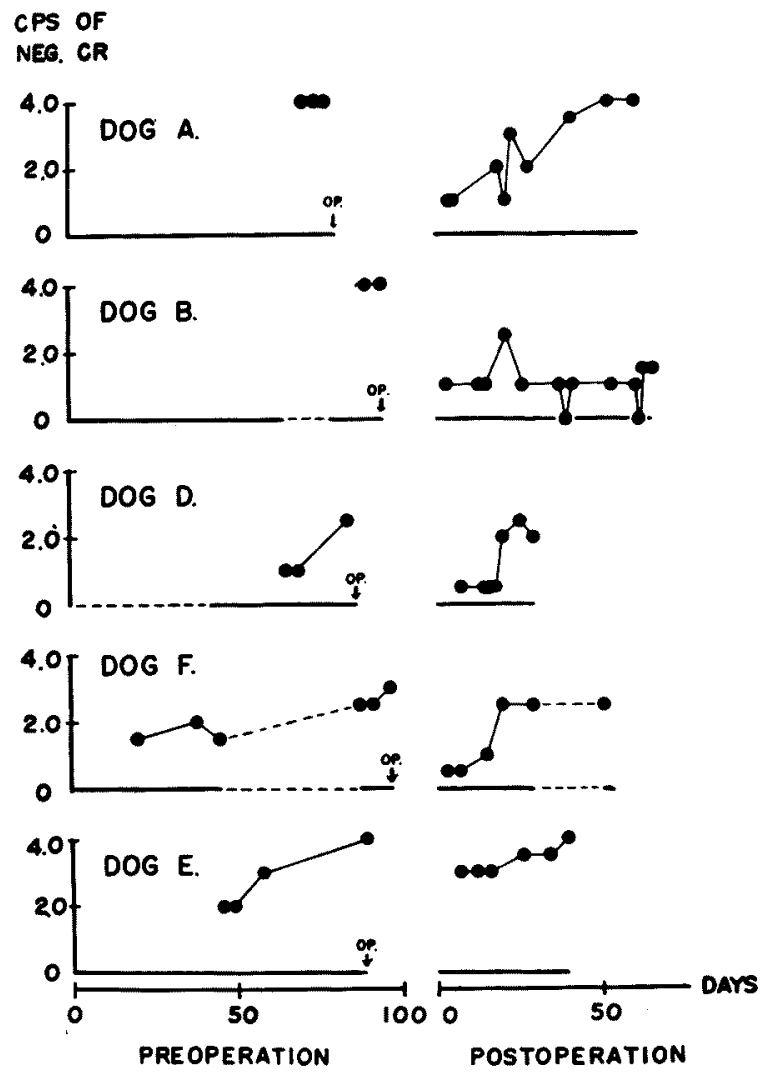

Fig. 8. Discrimination capacity at various pre-(left) and post-(right) operative stages. The higher flicker frequency serving as a pos. CS was fixed, and the frequency of neg. CS was varied. The highest frequency of neg. CS discriminated from pos. CS was plotted against days of training. Dashed lines of abscissae in Dogs $B$ and $F$ denote period of quiescense of training for retention tests. Dashed line in Dog $\mathrm{D}$ indicates period of training with problem $a$, which this dog could not learn.

field. It has often been reported that little retrograde degeneration of the lateral geniculate body was observed in the temporal-operated animals, in addition to the absence of defects either in visual field or in acuity ${ }^{1,12)}$. Thus, the impairments mentioned above may be considered to be specific for the ablated inferotemporal areas, and this interpretation receives support from the fact that no such impairments occurred in the control E-dogs. There is some evidence that an interruption of the thalamo-cortical relation or ablation of the prestriate cortex affected little visual discrimination (Blum et al. ${ }^{1)}$, Chow $\left.{ }^{5}\right)$. Even if these 
systems had been damaged in our experiments, it would be difficult to interpret the impairments under consideration as due to the damages in these systems. It was reported that the $\mathrm{T}$-animals showed complete retention of conditioning behavior, so far as simple conditioning with various visual stimuli was concerned (Chow ${ }^{3)}$, Mishkin \& Hall ${ }^{11)}$, Pribram \& Mishkin $\left.{ }^{19}\right)$. In the presant experiments, it was represented that $\operatorname{Dog} C$, which could not solve the problem $\alpha$ before operation, showed a complete generalization form to CSs after operation and that the retention of preoperatively learned discrimination was relatively high when the easier problem $\beta$ was used. This suggests that the T-dogs might be nearly normal in simple conditioning which started before operation as in the earlier experiments. It is, however, to be noticed that initial learning started postoperatively was found very difficult, as shown above.

It is suggested in the literatures that different parts of the temporal lobe are not equipotential for visual discrimination capactity of animals. In the present experiments, polus temporalis and gyrus temporalis inferior were resected without involvement of the deep allocortical structures and such remarkable visual impairments as stated above have been obtained. The present results agree with earlier reports on monkeys suggesting that the ventrolateral surface of the temporal lobe mediates visual discrimination ${ }^{3,10,12)}$, while that no visual deficits were caused by removal of the anteromedial region of the temporal lobe or destruction of allocortical structures ${ }^{18}$. The question as to which is more essential to visual function, polus temporalis or gyrus temporalis inferior, is not yet settled (Mishkin ${ }^{10)}$, Pinto Hamuy et $a l .{ }^{17)}$ ).

Whether or not the disturbances produced by the inferotemporal ablation are modality-specific is also an open question (Blum et $a l .{ }^{11}$, Orbach et al. ${ }^{14)}$ ).

Dog $B$, which received the lesion of a large part of bilateral amygdalae in addition to the intended areas, displayed remarkable changes in affection. The other dogs in which lesion did not reach the deep allocortical regions showed only cognitive changes, but displayed no affective ones. This finding seems to support the well-known theory that allo- and neo-cortical structures are concerned with affective and cognitive functions respectively (Papez $\left.{ }^{15)}\right)$.

No permanent deficits could be observed in all but two dogs. Even these two dogs might show complete recovery if training had been continued beyond 1000 trials. The mechanism of recovery is not known at present.

\section{SUMMARY}

Based on instrumental conditioned avoidance responses, retention and acquisition of visual discrimination of flicker frequencies were studied in 15 dogs with surgical lesion limitted to the bilateral neocortecies of polus temporalis and gyrus temporalis inferior. In addition, the differential threshold of visual performance, i.e., the least difference of flicker frequencies to be discriminated 
was determined at various pre- and postoperative stages in order to investigate the capacity to form learning sets, i.e., learn how to learn. Histological control of the ablated areas was made by a conventional method.

The results obtained are as follows :

1. The retention of the preoperatively learned discrimination was markedly reduced by bilateral ablation of the inferotemporal cortex, while it was not affected in the control animals which underwent bilateral removal of gyrus ectosylvius or unilateral lesion of the inferotemporal lobe.

2. For the inferotemporal-operated dogs, learning was further more difficult than for the control dogs.

3. Impairments in retention and acquisition were more marked, when tested with more difficult tasks.

4. The differential threshold of visual performance was remarkably impaired by ablation of the inferotemporal lobes, but it recovered to the preoperative level after a long period of training. It was, however, not affected by the control operation.

5. The inferotemporal-operated dogs showed some kind of spatial disorientation, while the control dogs did not.

6. The significance of the inferotemporal lobe for visual performance was discussed.

The author wishes to thank Prof. K. Motokawa for his invaluable discussion and suggestion throughout the course of the experiment and the preparation of the manuscript.

\section{References}

1) Blum, Josephine, S., Chow, K.L. \& Pribram, K.H., J. Comp. Neurol., 1950, 93, 53.

2) Brady, J.V., Schreiner, L., Geller, I. \& Kling, A., J. Comp. Physiol. Psychol., 1954, 47, 179 .

3) Chow, K.L., ibid., 1952, 45, 109.

4) Chow, K.L., ibid., 1954, 47, 194.

5) Chow, K.L., Arch. Neurol. Psychiat., 1954, 71, 762.

6) Harlow, H.F., Psychol. Rev., 1949, 56, 51.

7) Iwai, E., Okuda, J. \& Ishitoya, T., Tohoku J. Exp. Med., to be published.

8) Klüver, H. \& Bucy, P. C., Arch. Neurol. Psychiat., 1939, 42, 979.

9) Lim, R.K.S., Liu, C.-N. \& Moffitt, R.L., A Stereotaxic Atlas of the Dog's Brain, 1960, Springfield, Charles C Thomas.

10) Mishkin, M., J. Comp. Physiol. Psychol., 1954, 47, 187.

11) Mishkin, M. \& Hall, M., ibid., 1955, 48, 97.

12) Mishkin, M. \& Pribram, K.H., ibid., 1954, 47, 14.

13) Orbach, J. \& Fantz, R.L., ibid., 1958, 51, 126.

14) Orbach, J., Milner, B. \& Rasmussen, T., Arch. Neurol., 1960, 3, 230.

15) Papez, J.W., Arch. Neurol. Psychiat., 1937, 38, 725.

16) Pasik, T., Pasik, P., Battersby, W.S. \& Bender, M.B., J. Comp. Neurol., 1960, 115, 89.

17) Pinto Hamuy, T., Santibañez, G., Gonzales, C. \& Vicencio, E., J. Comp. Physiol. Psychol., 1957, 50, 379.

18) Pribram, K.H. \& Bagshaw, M., J. Comp. Neurol., 1953, 99, 347.

19) Pribram, K.H. \& Mishkin, M., J. Comp. Physiol. Psychol., 1955, 48, 198.

20) Thomas, G.J. \& Otis, L.S., ibid., 1958, 51, 130.

21) Weiskrantz, L., ibid., 1956, 49, 381. 